\title{
Hypersensitivity to local anaesthetics
}

\author{
Alicja Grzanka ${ }^{1}$, Iwona Wasilewska ${ }^{2}$, Magdalena Śliwczyńska ${ }^{3}$, Hanna Misiołek ${ }^{4}$ \\ ${ }^{1}$ Chair and Clinical Department of Internal Diseases, Dermatology and Alergology in Zabrze, \\ Medical University of Silesia in Katowice, Poland \\ ${ }^{2}$ Academic Centre of Dentistry and Specialist Medicine in Zabrze, Medical University of Silesia in Katowice, Poland \\ ${ }^{3}$ Department of Anaesthesiology and Intensive Therapy in Zabrze Medical University of Silesia in Katowice \\ (Student Scientific Society), Poland \\ ${ }^{4}$ Department of Anaesthesiology and Intensive Therapy in Zabrze Medical University of Silesia in Katowice, Poland
}

\begin{abstract}
Using local anaesthetics in daily practice, particularly by anaesthesiologists and dentists, is related to the risk of adverse reactions. Therefore, monitoring of such reactions, carrying out detailed documentation (according to the chart proposed in this study) and conducting specialist examinations is of the greatest importance. There is a variety of adverse reactions that may occur during local anaesthesia procedures, with the intensity ranging from clinically insignificant to life-threatening reactions. The majority of concerns reported by clinicians are linked to the appearance of various hypersensitivity reactions, including anaphylaxis. Healthcare providers responsible for the administration of local aneasthetics should be able to detect hypersensitivity reactions in order to implement appropriate treatment and then choose diagnostic procedure. The final diagnosis should be based on detailed history, medical record, including a case description and measurements of tryptase activity, skin tests and provocation trials. Screening tests are not currently recommended in individuals without hypersensitivity to local anaesthestics in history.
\end{abstract}

Key words: hypersensitivity; local anestehtics; local anesthesia; skin tests

Anaesthesiology Intensive Therapy 2016, vol. 48, no 2, 128-134

Introducing local aneasthetics (LAs) into daily clinical practice has entirely revolutionized operational procedures. In 1884, Austrian physician Carl Koller successfully superficially anaesthetized the cornea before an ophthalmology procedure using cocaine. Currently, LAs are widely used in a number of medical fields with a different range of their application possibilities. Such an increase in the number of local anaesthesia procedures must obviously have an impact on the increase of the possibility related to adverse drug reaction occurrence, including hypersensitivity reactions [1]. Adverse drug reactions resulting either from a pharmacological mechanism of action or the intensification of dose-dependent effects are usually well recognized and therefore predictable. In contrast, hypersensitivity reactions are still disputable, and their diagnosis poses a significant challenge to clinicians. Nevertheless, there has been some progress made over the last 2 years in this field. International expert guidelines have been published [2-5] with attention paid to the management of the problem related to drug hypersensitivity, which still remains one of the most enigmatic issues in allergology [6]. These recommendations also pertain to hypersensitivity reactions to LAs.

\section{DEFINITIONS}

The recommendations of Allergology and Anaesthesiology Scientific Societies $[2,4,7]$ on the management of drug hypersensitivity reactions consistently stress the necessity of using proper terminology. This enables efficient communication between clinicians, reliable research with a precise identification of recruited patients and the ability to compare the results obtained by particular investigators. This aspect is of special importance in clinical trials on unexpected adverse drug reactions which, for objective reasons, are based mainly on meta-analyses of case reports, as it is difficult to plan randomized, controlled trials in this field. 
Unexpected adverse drug reactions, the occurrence and intensity of which is dose-independent should be termed hypersensitivity reactions. The guidelines define them as objective, repeatable symptoms, elicited by exposure to certain drugs in well tolerated doses by the majority of patients. Drug allergy can be diagnosed only in situations when underlying immune mechanisms are documented in the development of hypersensitivity reactions i.e. IgEdependent or IgE-independent (most frequently T cell-dependent). In the remaining cases when other causes initiate the symptoms, nonallergic hypersensitivity is diagnosed. Anaphylaxis, the most serious form of hypersensitivity, is defined as a severe, life-threatening, generalized or systemic hypersensitivity reaction. It is divided into allergic (documented participation of immune mechanisms) and non-allergic (previously defined as anaphylactoid reaction) [8]. Hypersensitivity reactions are also classified as acute (immediate) and delayed (non-immediate). This classification does not consider pathomechanism of the reaction but the time from drug administration to the onset of clinical symptoms. Immediate hypersensitivity reactions, both allergic and non-allergic, occur within 0 to 6 hours (rarely within 6-12 hours and no longer than 24 hours) following the administration of LAs and have similar clinical manifestations (urticaria, Quincke's oedema, bronchospasm, rhinitis, conjunctivitis, gastrointestinal symptoms, and anaphylaxis, including anaphylactic shock). Delayed reactions occur within 24 to 72 hours after exposure (rarely after 6 hours) and manifest most frequently with maculopapular rash and delayed urticaria [2].

\section{EPIDEMIOLOGY}

The accurate incidence of adverse reactions due to LAs and their underlying mechanisms is difficult to assess. The International Classification of Diseases (ICD-10) does not facilitate it since no consideration is given to all the aspects of the present issue and it is directed towards diagnostic information. Additionally, not all cases are reported. According to some investigators, symptoms of hypersensitivity (urticaria/ Quincke's oedema, anaphylaxis) are the third most common adverse reaction due to LAs [9].

IgE-dependent reactions are the least frequently observed adverse reactions. The meta-analysis of all reports published in English language journals (from 1951 to 2011) confirmed the incidence of such cases (documented based on diagnostic procedures) to be $1 \%$ among patients with a suspicion of hypersensitivity to LAs [10]. Cell-mediated allergic reactions are more common whereas symptoms of non-allergic hypersensitivity are the most frequent [11]. However, the majority of adverse reactions, some of which mimic hypersensitivity reactions, are connected with other causes such as toxicity, the effect of concomitant adrenaline administration, vasovagal syncope, anxiety responses or hyperventilation syndrome [10,12].

\section{MANAGEMENT OF HYPERSENSITIVITY TO LOCAL ANAESTHETICS}

According to the World Allergy Organization (WAO), unexpected adverse reactions that occur during anaesthesia should be analysed and they constitute an indication for intensified diagnostic procedure and for consultations with specialists, including allergologists (Fig. 1 presents the proposed management algorithm). Local anaesthetics are essential to perform a number of procedures and they cannot be eliminated without explanation as regards their tolerance (approach suggested by some authors) [13, 14].

The diagnosis of unexpected adverse reactions during local anaesthesia is a difficult issue. The occurrence of these symptoms does not necessarily indicate hypersensitivity to LAs. Thus, attention is paid to the collaboration between allergologists (who have the preparation and skills required to diagnose hypersensitivity) and anaesthesiologists (who have the most experience in evaluating adverse reactions due to anaesthetics, resulting from their pharmacological and toxic characteristics) [7].

\section{Until the aetiology of unexpected adverse reactions is explained, the term hypersensitivity to local anaes- thetics should be used.}

When a patient presents or has previously presented with the symptoms suggestive of-allergy to LAs, it is recommended to use the term hypersensitivity to local anaesthetics in verbal and written communication until the aetiology is fully accounted for. A preliminary diagnosis of hypersensitivity to LAs (in the case of unexpected adverse reactions) is justified not only factually but also practically. Physicians administering local anaesthesia or referring patients to allergologists are not obligated to verify whether the causes of the symptoms are immune (allergic hypersensitivity) or non-immune (non-allergic hypersensitivity) mechanisms. For objective reasons, the diagnostics of hypersensitivity should cover a broader spectrum than allergy to LAs alone. It is important not to use the term allergy during conversations with patients until it is finally confirmed. Patients who report the diagnosis of allergy to other physicians may be wrongly considered to be allergic to a particular drug. Clinical practice shows that in some patients certain LAs have been avoided for many years based exclusively on vasovagal symptoms. Therefore, the crucial issue is information on the event delivered by a physician in the form of a discharge abstract and then given to a patient and attached to medical record when the patient is referred for further diagnostic procedure. These issues are discussed below. 


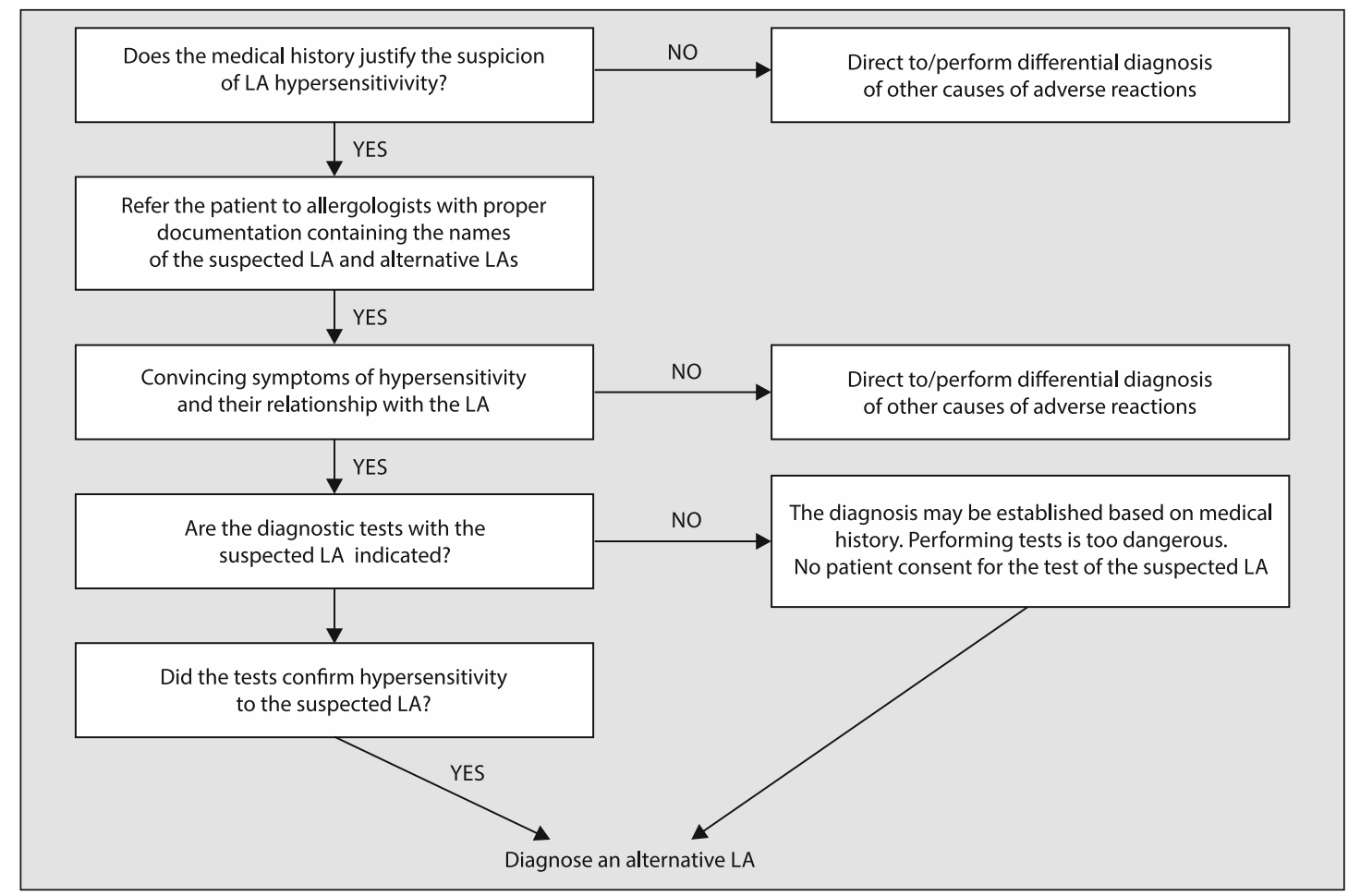

Figure 1. Management algorithm in case of suspicious of hypersensitivity to local anesthetics

2. Physicians administering the anaesthetic or those taking care of a patient during the occurrence of adverse reactions should make every effort to explain these reactions [7].

These actions should cover a reliable description of the event included in the discharge abstract given to a patient and also included in a referral for a consultation with a particular specialist with the attached medical record.

Information that should be provided in the patient documentation is as follows:

a) information on the drug suspected of causing the hypersensitivity reaction

(the trade name, dose, date, time and route of administration, previous tolerance to the drug or other drugs from this group if used before);

b) information on the hypersensitivity reaction

(date and time of symptom occurrence with their duration, a detailed description of clinical symptoms with special consideration given to the targeted organ or the system and photographic documentation if justified, management, diagnostic examinations, including laboratory tests);

Monitoring tryptase activity is particularly useful in differential diagnostics of the causes of adverse reactions (especially if anaphylaxis is suspected). Blood sample should be obtained 3 times i.e. immediately after the event and then 1-2 hours after symptom occurrence, as well as 24 hours after symptom resolution (with no detriment to the therapy). To do so, 5-10 mL of blood should be collected into a tube with a clot activator and centrifuged, and the serum should be refrigerated (if the evaluation is possible within 48 hours) [7] or frozen at a temperature of $-20^{\circ} \mathrm{C}$ [16]. An increased activity of tryptase is suggestive of anaphylaxis or another hypersensitivity reaction with mastocyte degranulation. The highest values are observed between 60 and 90 minutes after the onset of symptoms, after 6 hours tryptase activity is normalized and baseline values are obtained usually after 24 hours (baseline activity). Tryptase activity within the normal range does not exclude anaphylaxis. However, its increased value may retrospectively support the diagnosis of anaphylaxis. Despite the fact that the guidelines recommend monitoring serum tryptase activity in each suspected case of drug hypersensitivity reaction, these tests are too rarely performed.

In some cases with skin manifestations of hypersensitivity, a skin biopsy may be obtained for histological examination.

\section{c) additional information}

The trade names of other drugs, administered concomitantly with a "suspected" LA, should be included. Comorbidities, including allergic diseases, as well as symptoms of the infection, e.g. patient-reported dyspnoea could be a symptom of asthma existing for many years. Information on all previously suspected drug hypersensitivity reactions should be provided. 


\section{Is allergology consultation necessary before the ad-} ministration of local anaesthetics?

The current level of knowledge does not allow clinicians to predict which patients will present with a hypersensitivity reaction to LAs. Only the previous appearance of unexpected adverse reactions following the administration of LAs may be a risk factor of a similar or even more severe reaction after another exposure to the same agent. Therefore, a detailed history of previous tolerance to LAs should be the basis for making a decision about the possible use of a certain drug or patient referral to an allergologist. Patient evaluation before anaesthesia should also include the tolerance to other concomitantly administered agents (preservatives, antiseptic preparations, non-steroidal antiinflammatory drugs, antibiotics), as well as the materials and equipment with which patients may have contact during the procedure.

A history showing previous hypersensitivity reaction to LA administration should be included in the above-mentioned medical record and should result in a consultation with a specialist. Additionally, an allergological consultation confirming hypersensitivity to a certain local anaesthetic should also indicate an alternative drug that is safe to a patient. Patients without such medical record must be consulted by allergologists before the administration of a LA [17]. The referral is accompanied by not only a detailed description of the event but also information on the trade name of the planned LA (of first and second choices). At times allergologists are provided with a referral with a diagnosis of drug allergy and a request for a drug test.

Medical history is an important part of the allergological diagnostic procedure. The detailed description of the event may be so suggestive that allergologists may consider performing a particular drug test redundant and/or dangerous. The obvious causative relationship between anaphylaxis symptoms and a certain drug will allow the initiation of the diagnostic procedure starting from the assessment of tolerance to alternative drugs.

The retrospective evaluation of symptoms based only on patient history may be burdened with a large subjective error due to a sense of fear of patients at the time of the event and the time that has passed since the particular event. Moreover, patients are unable to present the majority of information necessary for a complete allergological evaluation. Therefore, a detailed history is of the greatest importance, together with event description provided by clinicians, including the information compatible with point 2 or the questionnaire proposed by the European Network for Drug Allergy (ENDA) [18]. Physicians administering anaesthetics or caring for patients during the occurrence of adverse drug reactions should enclose medical record con- taining the above-mentioned information when referring patients for an allergological consultation.

\section{Allergology procedures}

\section{a) skin tests}

Skin tests are an important part of the diagnostic procedure of allergic hypersensitivity to drugs. Nevertheless, in a number of cases, they have a limited diagnostic value due to the lack of standardized concentrations or an unknown predictive value of the results. However, optimal concentrations for skin tests were explicitly defined in the case of LAs, and their negative results have a significantly predictive value of up to $97 \%$ [19]. Thus, the evaluation of allergic hypersensitivity to LAs using these tests may be of practical importance, as they may either confirm or exclude allergy to LAs [5]. To perform the tests, commercially available drugs are used that do not contain adrenaline or other ingredients such as sulfites or parabens [20]. The following tests are used: skin prick tests (SPTs), intradermal tests (IDTs), and patch tests (PTs).

Prick tests have a higher specificity but a lower sensitivity and are connected with the lowest risk of anaphylaxis. They can be terminated at any time during the procedure. Nondiluted LA solutions are recommended (strong recommendation/high quality of evidence according to The Grading of Recommendations Assessment, Development and Evaluation (GRADE) [5, 21]).

Intradermal tests are more sensitive and repeatable, however they have a lower specificity and are related to a higher risk of adverse reactions. They are performed only after negative SPT results. Local anaesthetics are used at a dilution of 1: 10 (strong recommendation/high quality of evidence according to the GRADE [5]). It is recommended to do the test with gradually increasing concentrations (1: $1000,1: 100,1: 10$ ), which decreases the risk of serious adverse reactions [2].

Prick tests and IDTs detect IgE-dependent mechanisms (i.e. immediate reactions). However, in the diagnosis of LA hypersensitivity, they may be useful in some patients in detection of delayed reactions. For this reason, patients should be advised to observe the skin region subjected to tests for a prolonged period of time and to promptly inform the physician of any changes. Brockow et al. [5] recommend IDTs with LAs at a dilution of $1: 10$ equally to patch tests for the assessment of delayed allergic reactions. The delayed assessment of IDT results (measurement of the infiltration diameter after 48 hours) compared to PTs is of comparable or even higher sensitivity in the diagnosis of these reactions.

Patch tests are performed with undiluted drugs (strong recommendation/high quality of evidence according to the GRADE [5]). They allow the diagnosis of cell-mediated hypersensitivity and are useful in the diagnosis of contact 
Table 1. Reverse placebo provocation test $[2,26]$

\begin{tabular}{llll}
\hline Procedure & Information given to patient & Result of provocation & Recommended proceeding \\
\hline LA injection & LA injection & Patient reports complaints & Continuation of procedure \\
Saline injection & Next dose of LA injection & Patient reports complaints & Continuation of procedure \\
LA injection & Placebo injection & Patient does not report complaints & Continuation of procedure \\
& & Patient reports complaints & Positive result of provocation test \\
LA injection & $\begin{array}{l}\text { Informing the patient about previous } \\
\text { procedures; LA injection afterwards }\end{array}$ & Patient does not report complaints & Negative result of provocation test \\
\hline
\end{tabular}

allergies to LAs. The usefulness of PTs with LAs has been stressed [22]. Cell-mediated hypersensitivity is common with the use of ester compounds but is also possible with amide derivatives [23]. After using benzocaine to decrease pain at the injection site, symptoms of contact allergy may develop, sometimes erroneously interpreted as inflammatory infiltration [24, 25].

b) provocative tests (single-blind, placebo controlled)

Hypersensitivity to LAs is diagnosed independently of its mechanism with the use of provocative tests. These tests diagnose non-allergic hypersensitivity and confirm tolerance to LAs indicated in skin tests.

They should be always performed at the time when an alternative drug is chosen (strong recommendation/ high quality of evidence according to the GRADE [5]). Provocative tests with drugs are done only if possible benefits outweigh risks connected to them. The latter situation concerns LAs because they are hardly avoidable in a number of medical procedures [5]. The test is performed with a single-blind method (patients are not aware whether they are given a LA or placebo) to exclude unspecific or psychogenic reactions. The test begins with saline administration and then $0.1,0.2$, $0.5,1.0$, and $2.0 \mathrm{~mL}$ of undiluted LA (without adrenaline) is given subcutaneously into the extensor forearm region at the width of a hand over the elbow joint with time intervals of 30 minutes [26]. Patients should be informed in detail about the aim of the test and the risk involved as well as about the details of the procedure and placebo usage prior to written informed consent.

All guidelines unanimously emphasize that provocative tests should be the last stage of diagnostic procedure with negative skin test results. However, if allergological diagnosis cannot be conducted and the history is not explicit, provocative tests with an alternative drug without skin tests may be performed [27]. In these cases, undiluted LA (without adrenaline) is administered subcutaneously in doses of between $0.5 \mathrm{~mL}$ and $1 \mathrm{~mL}$. Lack of a hypersensitive reaction within 30 minutes after the injection is considered a negative result [16].

If symptoms occur during placebo administration, a reverse provocative test with placebo may be used. The following consecutive stages of the test are presented in Table 1.

\section{WHERE, WHEN AND IN WHOM IS DIAGNOSTIC PROCEDURE PERFORMED?}

Diagnostic procedure (including SPTs which are considered the safest) should be performed by experienced allergologists or done in specialized sites using typical precautions for allergological procedures.

Allergological diagnostics should be performed in patients in whom hypersensitivity symptoms occurred within 4 weeks up to 6 months after symptom resolution and the cessation of anti-allergic treatment. After this period, test results are less credible. It was shown that sensitivity to drug skin tests declines over time [2].

An allergological evaluation should also be indicated in the case of elective surgical procedure with local anaesthesia in patients who presented with symptoms of hypersensitivity in the distant past but do not have medical record supporting that event. Also, the aim of the evaluation is to indicate the drugs that are safe within this group. The anaesthesiologist should make every effort to obtain the information on the previous anaesthesia and deliver it to the allergologist [17].

In the case when clinical symptoms of hypersensitivity during local anaesthesia were unambiguous and the diagnostic procedure was negative, another diagnostic approach is recommended after approximately $4-6$ weeks. The reason for it is a suspicion of a time-related decreased ability to react to $L A s$ and the performed diagnostic evaluation may reactivate a state of hypersensitivity (booster effect).

In turn, performing the above allergological diagnostic procedure is not indicated in patients in whom the history did not show hypersensitivity symptoms after the use of LAs [2]. This also applies to patients with asthma and other atopic diseases and to patients with allergy to substances or drugs to which they will not be exposed during the procedure. Additionally, patients with mastocytosis who are particularly predisposed to anaphylaxis (especially drugrelated) do not comprise the risk group of adverse reactions after LAs. So far there have been only single case reports of hypersensitivity to LAs described in this group, despite the fact that each patient underwent bone marrow aspiration during local anaesthesia. Due to a lack of such reports, it is believed that the risk of hypersensitivity to LAs in patients 
with mastocytosis is not higher as compared to the general population [3].

Allergological diagnostic evaluation is not useful either in cases of adverse reactions resulting from pharmacological and toxic characteristics of LAs.

\section{CROSS-REACTIVITY}

Each case of documented hypersensitivity to a particular drug is related to the necessity of assessing whether there is a cross reactivity with other LAs available on the market. Additionally, an alternative safe drug must be selected. Good tolerance to the alternative drug should be confirmed not only on the basis of negative skin test results but also on the basis of provocative test results. However, the assumption of cross reactivity without medical record during the diagnostic procedure is a mistake. Cross reactions are typical of ester forms and are less frequently connected with amide derivatives. There are cases of allergic reactions to lidocaine with good tolerance to articaine [28] or symptoms of urticaria connected only to mepivacaine but with a lack of adverse reactions after the administration of lidocaine and bupivacaine [29]. However, the literature provides the data on concomitant allergy to a few drugs of amide derivatives [30]. The most common is cross reactivity between lidocaine and mepivacaine as indicated by French authors [31].

Good tolerance to an alternative LA in provocative tests (with hypersensitivity to others) does not allow the total exclusion of the participation of this drug in the future development of unexpected reactions. In such situations, premedication may be considered (administration of glucocorticoids and anti-histaminic drugs), which is more effective in symptom prevention of non-allergic hypersensitivity compared to IgE-dependent mechanisms (i.e. with symptoms more commonly related to LAs) [4]. Other authors use premedication despite the fact that although clear evidence concerning its efficacy is not available, the evidence connected with its inefficacy is also unavailable.

\section{OTHER CAUSES OF HYPERSENSITIVITY REACTIONS DURING LOCAL ANAESTHESIA PROCEDURES}

Local anaesthesia procedures are related to hypersensitivity not only to anaesthetics but also to preservative additives in particular drugs (parabens, sulfites) as well as latex and nickel or antiseptic preparations [32, 33]. Nickel may be released and absorbed by the organism by means of injection needles. Potential sources of latex include vials and cartridges containing LAs and syringes with latex caps and gloves. Preservative additives may be a cause of anaphylaxis or delayed reactions. Some reports indicate a significant participation of allergy to methylparaben in the development of hypersensitivity symptoms during local anaesthesia [10, 34]. Thus, diagnostic procedure connected with the reactions to preservative additives is recommended if symptoms of hypersensitivity occurred after the administration of LAs containing such preservatives. Consequently, the trade name of the LA is essential information that should be delivered to the consulting allergologist. Additionally, it has been stressed that during the diagnostic procedure of hypersensitivity to sulfites, only oral provocative tests are recommended (strong recommendation/moderate quality of evidence according to the GRADE) because both positive and negative skin test results have a low predictive value [5].

\section{SUMMARY}

Although hypersensitivity reactions account for a minority of all reported adverse reactions after the administration of LAs, they will occur proportionally to the frequency of the use of these drugs. Physicians administering LAs should know how to diagnose symptoms of hypersensitivity in order to introduce appropriate treatment and initiate activities that could allow future proper diagnostic evaluation. According to the recommendations, a detailed event description is of the greatest importance [2]. The occurrence of unexpected adverse effects during previous anaesthesia is the most important risk factor of their development after the administration of LAs. Vigilance is required even with a history of good tolerance to LAs because a hypersensitivity reaction may occur during the next exposure. A comprehensive diagnostic procedure of hypersensitivity to LAs should consider the data from objective medical record. Also, it should involve tests, allowing the assessment of immediate and delayed reactions, not only to LAs alone but also to the other drug ingredients and agents connected with anaesthesia [35].

\section{ACKNOWLEDGEMENTS}

1. The authors declare no financial disclosure.

2. The authors declare no conflict of interest.

\section{References:}

1. Joint Task Force on Practice Parameters; American Academy of Allergy, Asthma and Immunology; American College of Allergy, Asthma and Immunology; Joint Council of Allergy, Asthma and Immunology. Drug allergy: an updated practice parameter. Ann Allergy Asthma Immunol. 2010; 105: 259-273. doi: 10.1016/j.anai.2010.08.002.

2. Brockow K, Przybilla B, Aberer Wet al.: Guideline for the diagnosis of drug hypersensitivity reactions. Allergo J Int. 2015; 24: 94-105.

3. Bonadonna $\mathrm{P}$, Pagani $\mathrm{M}$, Aberer $W$ et al.: Drug hypersensitivity in clonal mast cell disorders: ENDA/EAACI position paper. Allergy 2015; 70: 755-763. doi: 10.1111/all.12617

4. Demoly P, Adkinson NF, Brockow Ket al.: International Consensus on drug allergy. Allergy. 2014; 69: 420-437. doi: 10.1111/all.12350.

5. Brockow $K$, Garvey $L H$, Aberer $W$ et al: Skin test concentrations for systemically administered drugs - an ENDA/EAACI Drug Allergy Interest Group position paper. Allergy 2013; 68: 702-712. doi: 10.1111/all.12142.

6. Leung DYM, Nelson HS, Szefler SJ, Busse WW: Drug hypersensitivity: beyond the hapten concept. J Allergy Clin Immunol 2004; 113: 372 . 
7. Harper $N J$, Dixon $T$, Dugué $P$ et al.: Suspected anaphylactic reactions associated with anaesthesia. Anaesthesia 2009; 64: 199-211. doi: 10.1111/j.1365.2044.2008.05733.x.

8. Johansson SG, Bieber T, Dahl R et al.: Revised nomenclature for allergy for global use: Report of the Nomenclature Review Committee of the World Allergy Organization, October 2003. J Allergy Clin Immunol. 2004; 113: 832-836. doi: 10.1016/s0091-6749(04)00930-3.

9. Fuzier R, Lapeyre-Mestre M, Samii K, Montastruc JL; French Association of Regional Pharmacovigilance Centres: Adverse drug reactions to local anaesthetics: a review of the French pharmacovigilance database. Drug Saf 2009; 32: 345-356. doi: 102165/00002018-200932040_00008.

10. Bhole MV, Manson AL, Seneviratne SL, Misbah SA: IgE-mediated allergy to local anaesthetics: separating fact from perception: a UK perspective. $\mathrm{Br}$ J Anaesthesia 2012; 108: 903-911. doi: 10.1093/bja/aes348.

11. Gunera-Saad N, Guillot I, Cousin F et al.: Immediate reactions to local anesthetics: diagnostic and therapeutic procedures. Ann Dermatol Venereol 2007; 134: 333-336.

12. Rood JP: Adverse reaction to dental local anaesthetic injection - 'allergy' is not the cause. Br Dent J 2000; 189: 380-384. doi: 10.1038/ sj.bdj.4800776a.

13. Gruchalla RS: Drug allergy. J Allergy Clin Immunol 2000; 105: 637-644. doi: 10.1067/mai.2000.106156.

14. whttp://www.worldallergy. org/professional/allergic diseases

15. Harper $N J$, Dixon $T$, Dugué $P$ et al.: Suspected anaphylactic reactions associated with anaesthesia. Anaesthesia 2009; 64: 199-211. doi: 10.1111/j.1365-2044-2008.05733.x.

16. Mertes PM, Malinovsky JM, Jouffroy L et al.: Reducing the risk of anaphylaxis during anesthesia: 2011 updated guidelines for clinical practice. J Investig Allergol Clin Immunol 2011; 21: 442-453.

17. DeHert S, Imberger G, Carlisle J et al.: Preoperative evaluation of the adult patient undergoing non-cardiac surgery: guidelines from the European Society of Anaesthesiology. Eur J Anaesthesiol. 2011; 28: 684-722. doi: 10.1097/eja0b013e3283499e3b.

18. Bousquet $P-J$, Demoly $P$, Romano $A$ et al.: Pharmacovigilance of drug allergy and hypersensitivity using the ENDA-DAHD database and the GALEN platform. The Galenda project. Allergy 2009; 9: 194-203. doi: 10.1111/j.1398-9995.2008.01944.x.

19. McClimon B, Rank M, Li J: The predictive value of skin testing in the diagnosis of local anaesthetic allergy. Allergy Asthma Proc. 2011; 32: 95-98. doi: 10.2500/aap.2011.32.3417.

20. MacyE, Schatz M, Zeiger RS: Immediate hypersensitivity to methylparaben causing false-positive results of local anesthetic skin testing or provocative dose testing. Permanente J 2002; 6: 17-21.

21. Atkins $D$, Best $D$, Briss $P A$ et al.: Grading quality of evidence and strength of recommendations. BMJ 2004; 328: 1490. doi: 10.1136/ bmj.328.7454.1490.

22. Brinca A, Cabral R, Goncalo M: Contact allergy to local anaestheticsvalue of patch testing with a caine mix in the baseline series. Contact Dermatitis 2013; 68: 156-162. doi: 10.1111/j.1600-0536.2012.02149.x.

23. Klein CE, Gall H:Type IV allergy to amide-type local anesthetics. Contact Dermatitis 1991; 25: 45-48. doi: 10.1111/j.1600-0536.1991.tb01772.x.
24. Melamed J, Beaucher WN: Local anesthetic allergy characterized by type IV reaction in patients who received dental anesthesia J Allergy Clin Immunol 2006; 117: 225. doi: 10.1016/j.jaci.2005.12.888.

25. Fernández-Redondo $V$, León A, Santiago T: Allergic contact dermatitis from local anesthetic on peristomal skin. Contact Dermatitis 2001; 5: 358. doi: 10.1034/j.1600-0536.2001.450609.x.

26. Ring J, Franz K, Brockow K: Anaphylactic reactions to local anesthetics. Chem Immunol Allergy 2010; 95: 190-200. doi: 10.1159/000315952.

27. Chiriac AM, Demoly P: Drug provocation tests: up-date and novel approaches. Allergy Asthma Clin Immunol 2013; 9: 12. doi: 10.1186/17101492-9-12.

28. Al-Dosary K, Al-Qahtani A, Alangari A: Anaphylaxis to lidocaine with tolerance to articaine in a 12 year old girl. Saudi Pharm J 2014; 22: 280-282. doi: 10.1016/j.jsps.2013.10.001.

29. Prieto $A$, Herrero $T$, Rubio $M$ et al.: Urticaria due to mepivacaine with tolerance to lidocaine and bupivacaine. Allergy 2005; 60: 261-262. doi: 10.1111/j.1398-9995.2005.00648.x.

30. Cuesta-Herranz J, de las Heras M, Fernández $M$ et al.: Allergic reaction caused by local anesthetic agents belonging to the amide group. J Allergy Clin Immunol 1997; 99: 427-428. doi: 10.1097/01206501199712000-00018.

31. Fuzier R, Lapeyre-Mestre M, Mertes PM et al.: Immediate- and delayedtype allergic reactions to amide local anesthetics: clinical features and skin testing. French Association of Regional Pharmacovigilance Centers. Pharmacoepidemiol. Drug Saf 2009; 18:595-601. doi: 10.1002/ pds.1758.

32. Thyssen JP, Menné T, Elberling J, Plaschke P, Johansen JD: Hypersensitivity to local anaesthetics--update and proposal of evaluation algorithm. Contact Dermatitis 2008; 59: 69-78. doi: 10.1111/j.16000536.2008.01366.x.

33. Parkes AW, Harper N, Herwadkar A, Pumphrey R: Anaphylaxis to the chlorhexidine component of Instillagel(R): a case series. Br J Anaesth 2009; 102: 65-68. doi: 10.1093(bja)aen324.

34. Macy E, Schatz M, Zeiger RS: Immediate hypersensitivity to methylparaben causing false-positive results of local anesthetic skin testing or provocative dose testing. Permanente J 2002; 6: 17-21.

35. Grzanka A, Misiołek H, Filipowska A, Miśkiewicz-Orczyk K, Jarząb J: Nadwrażliwość czy alergia na środki znieczulenia miejscowego? Anestezjol Intens Ter 2010; 42: 175-178.

\section{Corresponding author:}

Prof. Hanna Misiołek. MD, PhD

Department of Anaesthesiology and Intensive Therapy

Faculty of Medicine with Division of Dentistry in Zabrze

Medical University of Silesia in Katowice

ul. 3-go Maja 13-15, 41-800 Zabrze, Poland

e-mail:hanna.misiolek@gmail.com

Received: 17.11.2015

Accepted: 2.01.2016 